\title{
Photonic crystal microcavities in SOI waveguides produced in a CMOS environment
}

Meister, Stefan, Al-Saadi, Aws, Franke, Bülent, Mahdi, Shaimaa, Kuhlow, Berndt, et al.

Stefan Meister, Aws Al-Saadi, Bülent A. Franke, Shaimaa Mahdi, Berndt Kuhlow, Karsten Voigt, Bernd Tillack, Harald H. Richter, Lars Zimmermann, Viachaslau Ksianzou, Sigurd K. Schrader, Hans J. Eichler, "Photonic crystal microcavities in SOI waveguides produced in a CMOS environment," Proc. SPIE 7606, Silicon Photonics V, 760616 (16 February 2010); doi: 10.1117/12.842714

SPIE. Event: SPIE OPTO, 2010, San Francisco, California, United States 


\title{
Photonic crystal microcavities in SOI waveguides produced in a CMOS environment
}

\author{
Stefan Meister*a , Aws Al-Saadi ${ }^{a}$, Bülent A. Franke ${ }^{\mathrm{a}}$, Shaimaa Mahdi ${ }^{\mathrm{a}}$, Berndt Kuhlow ${ }^{\mathrm{a}}$, \\ Karsten Voigt ${ }^{\mathrm{b}}$, Bernd Tillack ${ }^{\mathrm{c}}$, Harald H. Richter ${ }^{\mathrm{c}}$, Lars Zimmermann ${ }^{\mathrm{c}}$, Viachaslau Ksianzou ${ }^{\mathrm{d}}$, \\ Sigurd K. Schrader ${ }^{\mathrm{d}}$, Hans J. Eichler ${ }^{\mathrm{a}}$ \\ ${ }^{a}$ Technische Univ. Berlin, Institut für Optik und Atomare Physik, \\ ER1-1, Str. des 17. Juni 135, 10623 Berlin, Germany; \\ ${ }^{\mathrm{b}}$ Technische Univ. Berlin, Institut für Hochfrequenz- und Halbleiter-Systemtechnologien, HFT 4, \\ Str. des 17. Juni 135, 10623 Berlin, Germany; \\ 'Innovations for High Performance microelectronics (IHP) GmbH, Im Technologiepark 25, \\ 15236 Frankfurt (Oder), Germany; \\ ${ }^{\mathrm{d}}$ Technische Fachhochschule Wildau, Instituts für Plasma- und Lasertechnik, Bahnhofstr. \\ 15745 Wildau, Germany
}

\begin{abstract}
We have investigated microcavities in Silicon-on-Insolator (SOI) waveguides. The rectangular waveguides with $500 \mathrm{~nm}$ width are fabricated in the $220 \mathrm{~nm}$ silicon device layer. The microcavities are formed by one-dimensional photonic crystals in Fabry-Perot structure directly written in the waveguides.

The SOI photonic structures are produced in a CMOS environment using $248 \mathrm{~nm}$ DUV lithography, where the waveguides as well as the photonic crystals are created in the same step using a single mask.

In order to achieve a desired spectral shape of the filter function capable for several applications, a number of different cavities were investigated, e.g. single cavities of first and higher order as well as multi-cavity filters.

The experimental results are compared with simulations of photonic crystal microcavities in strip waveguides. The spectral transmission function of such filters dependent on the design parameters are calculated by an analysis based on Finite-Difference-Time-Domain (FDTD) method.
\end{abstract}

Keywords: silicon photonics, SOI-waveguides, photonic crystal, band pass filter, microcavity, FDTD simulation

\section{INTRODUCTION}

Silicon photonics, especially based on silicon-on-insulator (SOI) attracted great attention in the last years. With an intrinsic bandgap of $1.1 \mathrm{eV}$ silicon is transparent at wavelengths typically used for optical communication transmission, i.e. $1270 \mathrm{~nm}-1625 \mathrm{~nm}$. In addition, the possibility to produce silicon photonics devices in a complementary metal oxide semiconductor (CMOS) environment offers an opportunity for very low-cost photonic solutions. Furthermore, the ability to integrate silicon photonic devices with electronic control functions opens a wide range of applications from telecommunications to chip-to-chip interconnects ${ }^{1}$, on-chip communication as well as possible applications in emerging areas such as optical sensing and biomedical devices ${ }^{2}$.

Ultrasmall cavities play an important role in photonic integrates circuits (PIC) by controlling light for trapping and emission of photons ${ }^{3}$, lasing ${ }^{4}$, switching, and optical filtering. Most popular structures are ring resonators ${ }^{5}$ and photonic crystal microcavities. One-dimensional (1D) photonic crystal microcavities ${ }^{6,7}$ have a very small footprint and are, therefore, well suited for highly dense packaging.

*smeister@physik.tu-berlin.de; phone: +49-30-31426227; fax: +49-30-31426888

Silicon Photonics V, edited by Joel A. Kubby, Graham T. Reed, Proc. of SPIE Vol. 7606, 760616

(c) 2010 SPIE · CCC code: 0277-786X/10/\$18 · doi: 10.1117/12.842714

Proc. of SPIE Vol. 7606 760616-1 
In this paper, the characteristics of 1D photonic crystal microcavities on a SOI substrate are investigated, which are fabricated in a CMOS environment. The microcavities act as band pass filters. The filter design parameters, e.g. hole diameter, cavity length, cavity order, etc., are systematically changed to investigate the limites of such 1D photonic crystal microcavities. Furthermore, the behavior of multi-cavity filters is studied.

\section{DESIGN AND SIMULATION}

The filters here are designed for the telecommunications wavelength range around $1550 \mathrm{~nm}$ (including $\mathrm{C}$ and $\mathrm{L}$ Band). The microcavities are formed by one-dimensional photonic crystals in Fabry-Perot structure directly written in rectangular waveguides. Figure 1a shows the basic structure and parameters of the photonic crystal microcavities.
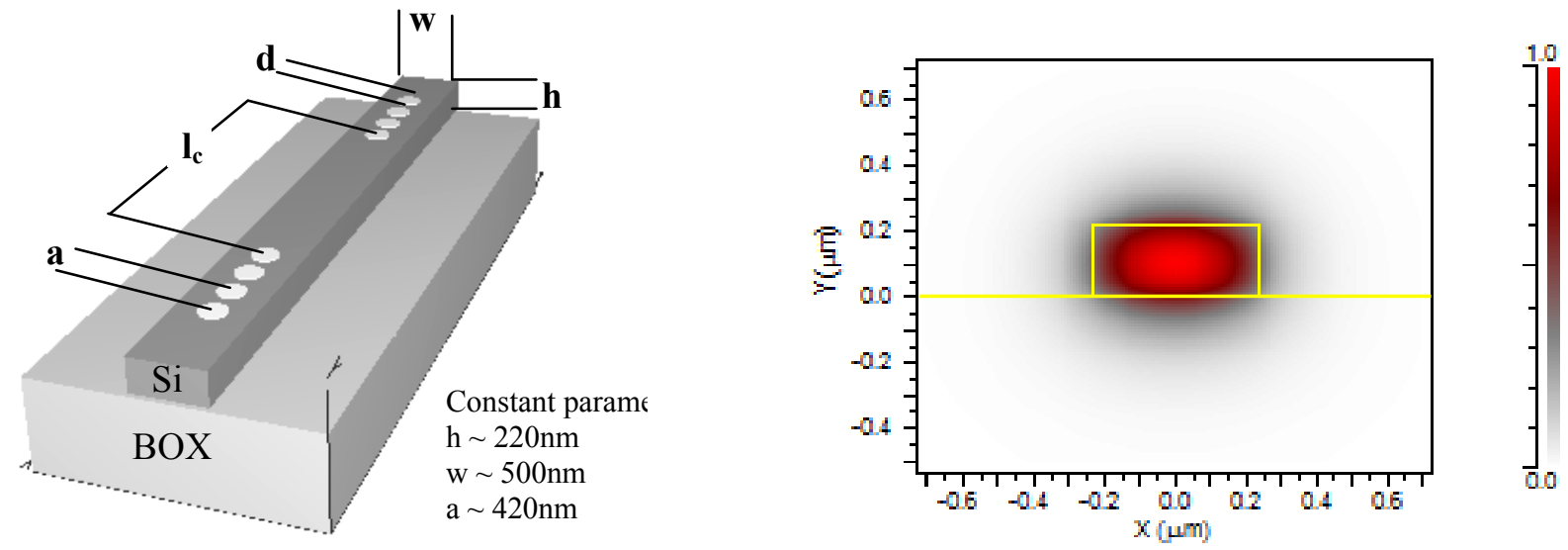

Figure 1. a) Basic structure and parameters of the photonic crystal microcavities. b) Mode field distribution in the SOI waveguide simulated by FullWAVE software.

The lattice constant a and cavity length $l_{c}$ are both measured from center to center between neighboring holes. The rectangular waveguides (also called nanowires) are fabricated in the $220 \mathrm{~nm}$ thick silicon device layer of the SOI substrate. Therefore, the waveguide hight is fixed to $h=220 \mathrm{~nm}$. Commonly, all the relevant geometric parameters are scaled with respect to only one parameter, e.g. the lattice constant a. Simply scaling the whole parameter set then allows matching to any desired wavelength ${ }^{8}$. The waveguide mode penetrates to some extent into the underlying $\mathrm{SiO}_{2}$ layer as seen in the simulated mode field in figure $1 \mathrm{~b}$. Therefore, the buried oxide (BOX) layer should have a thickness of at least $1 \mu \mathrm{m}$ to avoid waveguide leakage. In our case, the BOX layer has a thickness of $2 \mu \mathrm{m}$.

For a well-defined filter performance, a single-mode behavior of the utilized strip waveguide is aimed. Due to some performance and fabrication issues, a rectangular waveguide cross section is more favorable than a quadratic cross section $^{7}$. For the given waveguide height of $\mathrm{h}=220 \mathrm{~nm}$, a width of $\mathrm{w}=500 \mathrm{~nm}$ is suited to assure singlemode transmission in the telecommunication wavelength range around $1550 \mathrm{~nm}$. In this device geometry, the air-filled holes penetrate vertically through the wider waveguide side, as shown in figure 1a. TE polarization (E-field along the wider waveguide side and therefore perpendicular to the holes axes) is necessary to excite the cavity modes.

For optical testing requirements, the waveguides are extended on both sides of the photonic crystals by about $750 \mu \mathrm{m}$, and input and output ports are laterally displaced by $\pm 50 \mu \mathrm{m}$ in opposite directions via S-bends to prevent from light transmitted directly through the substrate ${ }^{7}$. The total waveguide length results then in about $1.5 \mathrm{~mm}$, where the photonic crystal microcavity is located in the central singlemode waveguide section, which is only about $100-\mu \mathrm{m}$ long. The waveguide is widened adiabatically to about $2 \mu \mathrm{m}$ on both sides of the photonic crystal region, and the input and output ends are again widened to $5 \mu \mathrm{m}$ in width in order to ease light coupling.

Different photonic crystal microcavities were designed and investigated in order to achieve the desired spectral shape of the filter function for several applications, for instances single cavities of first and higher order as well as multi-cavity filters. Furthermore, several design parameters were changed systematically to find out optimum settings. As a result, a large number of different photonic crystal microcavity filter structures have been implemented in our mask design. 
The fixed lattice constants $\mathrm{a}=420 \mathrm{~nm}$ have been chosen for all photonic crystal mirrors, see figure 1a. Also the waveguide width $\mathrm{w}$ was set to $500 \mathrm{~nm}$ in the design. Different hole diameters between $\mathrm{d}=200 \mathrm{~nm}$ and $\mathrm{d}=260 \mathrm{~nm}$ were realized in the mask design. The microcavities were designed with photonic crystal mirror pairs of 2 to 5 holes, e.g. $4+4$ holes. For the first order cavity filters, six different cavity lengths $1_{c}$ were introduced, ranging from $l_{c}=610 \mathrm{~nm}$ to $660 \mathrm{~nm}$ in steps of $10 \mathrm{~nm}$. The higher order cavities were designed in a symmetric structure, e.g. $3+6+3$ holes as shown in figure 2.

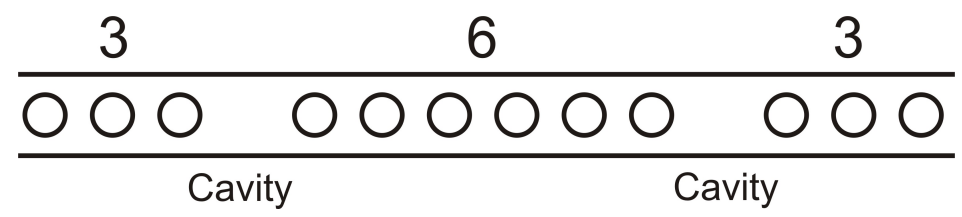

Figure 2. Structure of a photonic crystal double cavity filter with $3+6+3$ holes.

Simulation calculations are mandatory to specify the relevant parameters of photonic crystal microcavities in strip waveguides. The spectral transmission function of the filters dependent on the design parameters are calculated by an analysis based on Finite-Difference-Time-Domain (FDTD) method, using software "FullWAVE" from RSoft company.

3-D calculations were performed for these photonic crystal microcavity structures. The used grid size in $\mathrm{x}, \mathrm{y}$, and $\mathrm{z}$ was $0.025 \mu \mathrm{m}$ each, and the time step for satisfying the Courant stability conditions was $0.014 \mathrm{ct}$. The domain size was $\pm 0.7 \mu \mathrm{m}$ in $\mathrm{x}$ and $\mathrm{y}$. The method of pulse excitation and subsequent Fourier analysis of the transmitted pulse was used to calculate the spectral characteristics of the photonic crystal microcavities. All holes in a photonic crystal microcavity structure were set with a uniform diameter.

\section{FABRICATION OF PHOTONIC CRYSTALS}

The SOI photonic structures are produced in a $0.13 \mu \mathrm{m}$ SiGe BiCMOS environment at IHP GmbH, Frankfurt (Oder), Germany. The photonic devices were fabricated in $200 \mathrm{~mm}$ SOI wafers using a $248 \mathrm{~nm}$ DUV lithography. Waveguides as well as photonic crystal microcavities are designed using a single mask and fabricated in a shallow trench process.

Physically, the waveguides were formed by inserting two grooves into the SOI wafer surface running in parallel in the distance of the desired waveguide width. These lateral trenches are wide enough to prevent waveguide leakage into the surrounding intact top SOI areas.

$1.5 \mathrm{~mm}$ stripes containing rows of waveguides are then diced. Both diced facets are lapped and polished to allow the optical characterization of the photonic crystal microcavities.
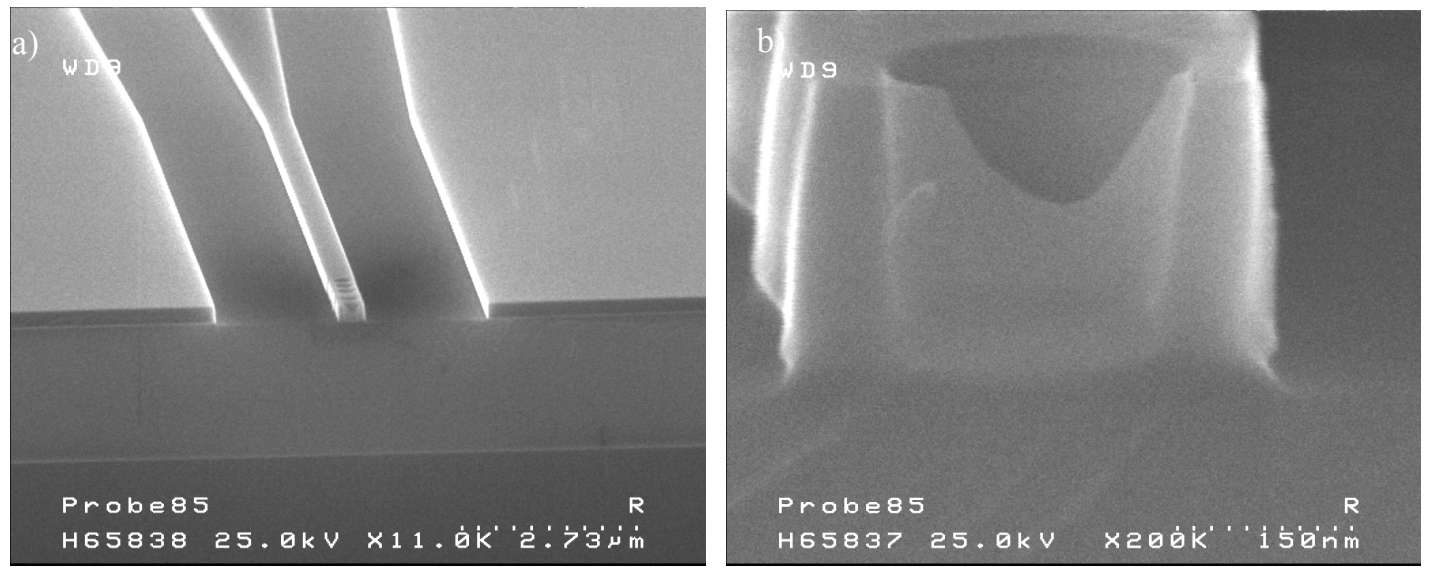

Figure 3. SEM image of a) a cross section through a SOI die with rectangular waveguide and b) a cross section through one hole of a photonic crystal mirror. 
Figure 3a shows a SEM image of a cross section through a SOI die with rectangular waveguide. Clearly seen are the two etched tranches in the $220 \mathrm{~nm} \mathrm{Si}$ top layer forming the waveguide. Also the BOX layer with a thickness of $2 \mu \mathrm{m}$ is seen. Figure $3 \mathrm{~b}$ shows the cross section through one hole of a photonic crystal mirror. It has a slightly conical shape and is etched completely down to the $\mathrm{SiO}_{2} \mathrm{BOX}$ layer. In this case, the hole has a diameter of $264 \mathrm{~nm}$ at the top. Also seen is a corrugation of the waveguide edges in the range of the holes forming the photonic crystals. Such corrugations appear when the hole diameters are larger than $230 \mathrm{~nm}$.

A complete $5+5$ photonic crystal microcavity is shown in figure 4a. In this case, the holes diameter is $210 \mathrm{~nm}$. Figure $4 \mathrm{~b}$ shows a SEM image of a waveguide with $220 \mathrm{~nm}$ hole in larger magnification. Both figures show a uniform shape of the holes and a nearly flat edge of the waveguide in the range of the holes.
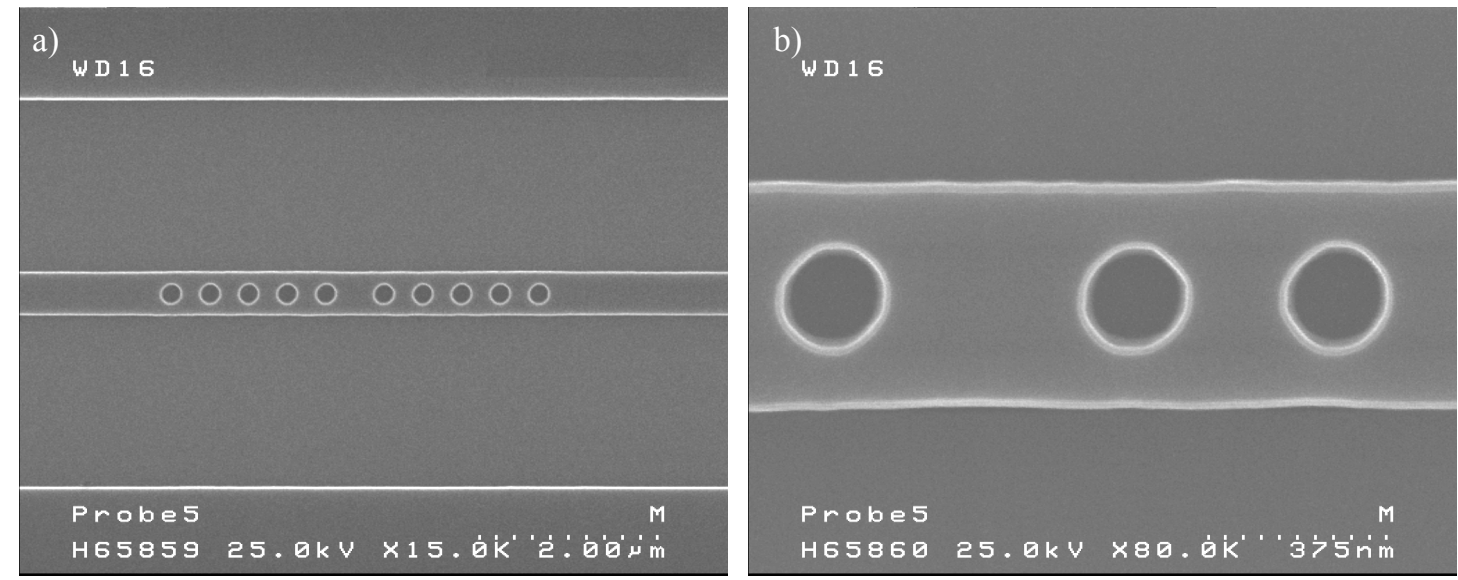

Figure 4. SEM image of a) a 5+5 photonic crystal microcavity filter etched in a SOI waveguide and b) holes with $220 \mathrm{~nm}$ diameter of a photonic crystal mirror in a SOI waveguide.

\section{EXPERIMENTAL DETAILS FOR OPTICAL CHARACTERISATION}

Figure 5a shows the experimental setup for optical characterization of the photonic crystal microcavities. The samples to be tested were fixed on a specially constructed vacuum chuck with integrated temperature control on top of a translation stage. A tunable external cavity laser (ECL) from Agilent 81940A served as light sources for transmission measurements. The wavelength band ranged from 1520 to $1630 \mathrm{~nm}$. The bandwidth of the laser is $<1 \mathrm{pm}$. Laser light was coupled into the SIO waveguides via a tapered optical fiber (OZ Optics), see figure 5b.

a)

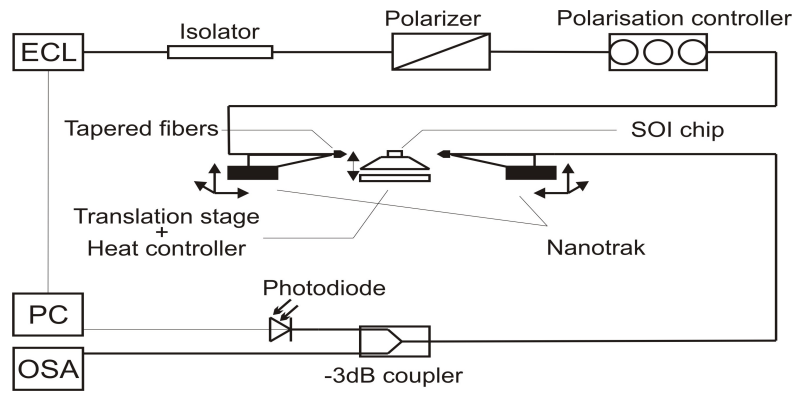

b)

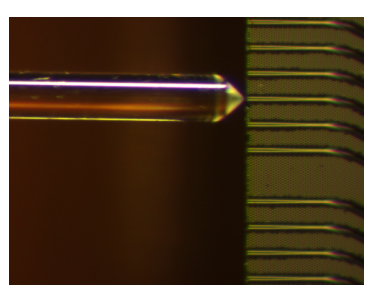

Figure 5. a) Experimental setup for optical characterization of the photonic crystal microcavities. b) Tapered fiber which couples the light in a SOI waveguide.

The taped fiber has a focal diameter of $2.5 \mu \mathrm{m}$ and a working distance of $14 \mu \mathrm{m}$. In order to achieve TE polarization at the waveguide input, a fiber optical polarizer followed by a polarization controller ${ }^{9}$ was inserted. The output light of the 
SOI waveguide was collected by a second (identical) tapered fiber. Both tapered fibers were mounted on a piezo controlled translation stage (NanoMax TS3), respectively. The output light was measured by a calibrated photo diode, which is part of the feedback control of the translation stage. A fraction of the output light was guided to an Optical Spectrum Analyzer (OSA) for online control of the output spectrum using the background signal of the ECL. The transmission spectrum of the photonic device was recorded by sweeping the laser wavelength.

\section{RESULTS AND DISCUSSTION}

The laser power at the input of the SOI waveguide was set to $1 \mathrm{~mW}$. As reference, dummy waveguides were used which had the same physical dimension as the waveguides carrying photonic crystal microcavities. The transmitted power in this case was typically $-20 \mathrm{dBm}$ or $10 \mu \mathrm{W}$, respectively, which can serves as a reference value. Most of the induced losses can be assigned to coupling losses from mode mismatch between the fibers and the SOI waveguide. It should be noted that the transmitted power is strongly dependent on the coupling conditions, which leads to a relatively high uncertainty in loss determination.

Usually, when measuring the filter transmission in a wavelength point-to-point mode, the transmission is superposed by high frequent Fabry-Perot resonances. In our case, these had a frequency of about $56 \mathrm{GHz}$, resulting from the waveguide cavities between the SOI facets and the photonic crystal mirrors in the center of the device. These resonances are rather disturbing and would also not appear in case of integrated filter components. Therefore, the spectral curves, measured by sweeping steps of $0.17 \mathrm{~nm}$, were averaged over 5 to 10 points. The original curves are seen in the background.

The experimental results are compared with the simulations of photonic crystal microcavities in strip waveguides. In the simulations, the necessarily chosen grid will introduce roughness to the holes, which would lead to scattering losses. Hence, the absolute value of these losses doed not fit exactly to the measured losses in the real waveguides. Therefore, the calculated peak transmissions of the filters differ from the measured one. But the influence of different parameters on the filter performance can be well analyzed by these simulations.

\subsection{First order single cavity}

In this part, the transmission of single cavity filters, formed by two identical photonic crystal mirrors, are investigated. Furthermore, the cavities are of the first order, which means the cavity length $l_{\mathrm{c}}$ has the shortest possible length for a certain transmission peak wavelength. In a classical Fabry-Perot, this would correspond to an optical spacer thickness of $\lambda / 2$, if $\lambda$ is the center wavelength at the transmission band of the filter. Figure 6 shows the transmission band of a photonic crystal microcavity filter with $4+4$ holes, in a) logarithmic and b) linear scale, respectively. The diameter of the holes was $\mathrm{d}=200 \mathrm{~nm}$ and the cavity length $1_{\mathrm{c}}=630 \mathrm{~nm}$.
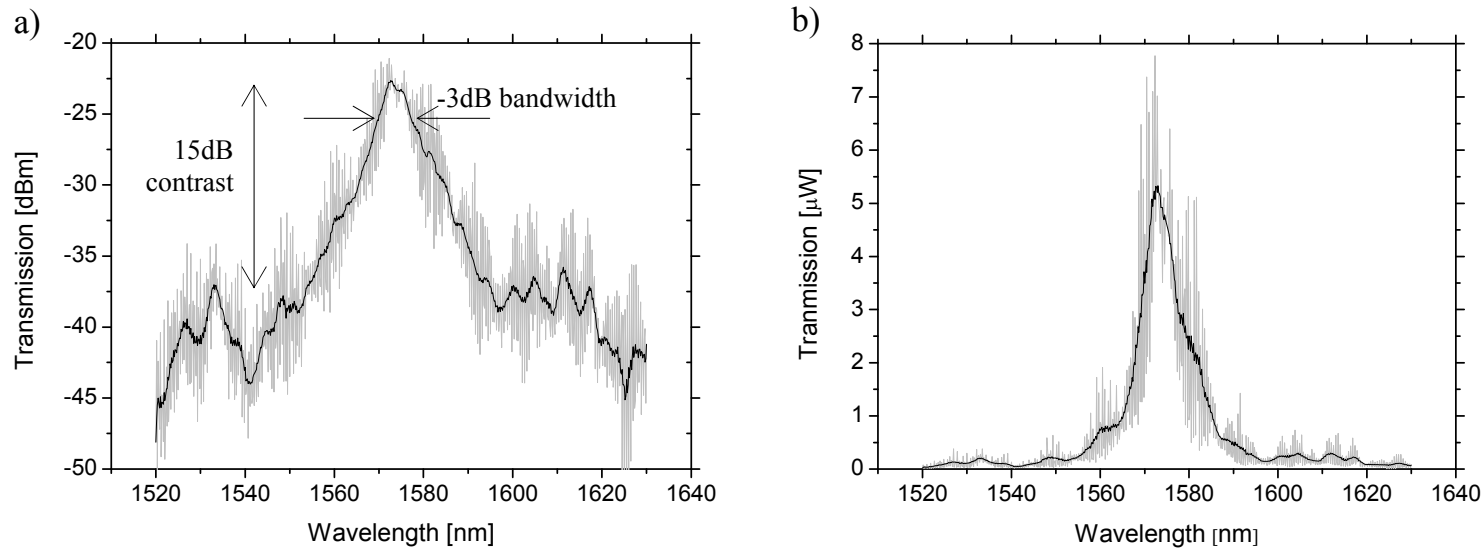

Figure 6. Transmission band of a photonic crystal microcavity filter with $4+4$ holes. a) Logarithmic scale, b) linear scale. The hole diameter is $200 \mathrm{~nm}$ and the cavity length is $1_{\mathrm{c}}=630 \mathrm{~nm}$. 
The $-3 \mathrm{~dB}$ bandwidth of the filter is $8.4 \mathrm{~nm}$. The filter contrast is about $15 \mathrm{~dB}$ for wavelengths $30 \mathrm{~nm}$ off to the filter peak. In insertion loss of the filter is $2.7 \mathrm{~dB}$.

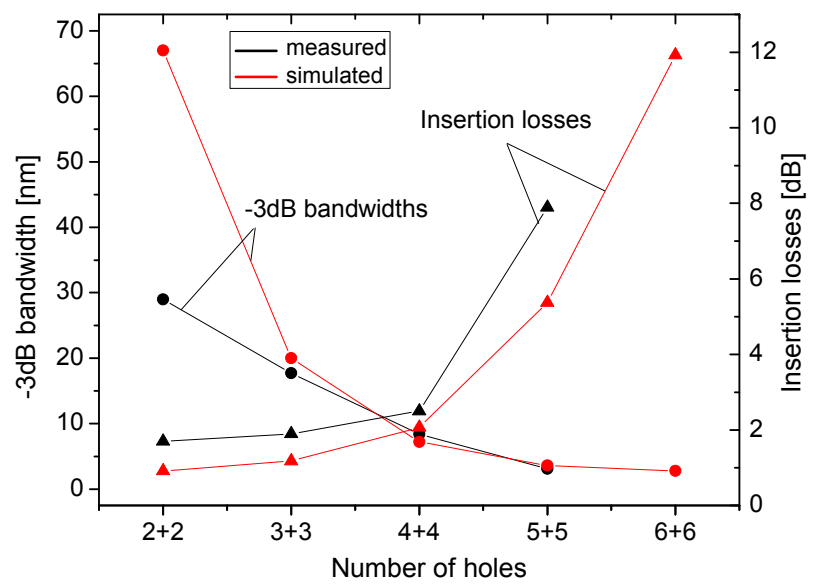

Figure 7. Measured and simulated $-3 \mathrm{~dB}$ bandwidths and insertion losses depending on the number of holes for first order single cavity filters. The hole diameter is $d=200 \mathrm{~nm}$ and the cavity length is $1_{c}=630 \mathrm{~nm}$.

Figure 7 shows the $-3 \mathrm{~dB}$ bandwidth as well as the insertion losses of microcavity filters depending on the numbers of holes. The $-3 \mathrm{~dB}$ bandwidth of the filters is strongly decreasing with the number of holes. That is obvious, because the number of holes in the photonic crystal mirrors on both sides of the microcavity dictates the mirror reflectivity, which is responsible for cavity mode confinement. With an increasing number of holes in the mirrors, an increasing reflectivity results and, therefore, a higher filter finesse arises. On the other hand, the insertion loss of the filters grow with the number of holes, as seen in figure 7. Since these losses limit the mode confinement also the maximum filter finesse is limited. Therefore, the minimal bandwidth becomes saturated by further increasing the number of holes. The hole diameter here was $\mathrm{d}=200 \mathrm{~nm}$, and the cavity length was $\mathrm{l}_{\mathrm{c}}=630 \mathrm{~nm}$.

For a photonic crystal microcavity containing $5+5$ holes a $-3 \mathrm{~dB}$ bandwidth of $3.1 \mathrm{~nm}$ was observed with gives a quality factor of $\mathrm{Q}=500$ for a center-wavelength of $1570 \mathrm{~nm}$.

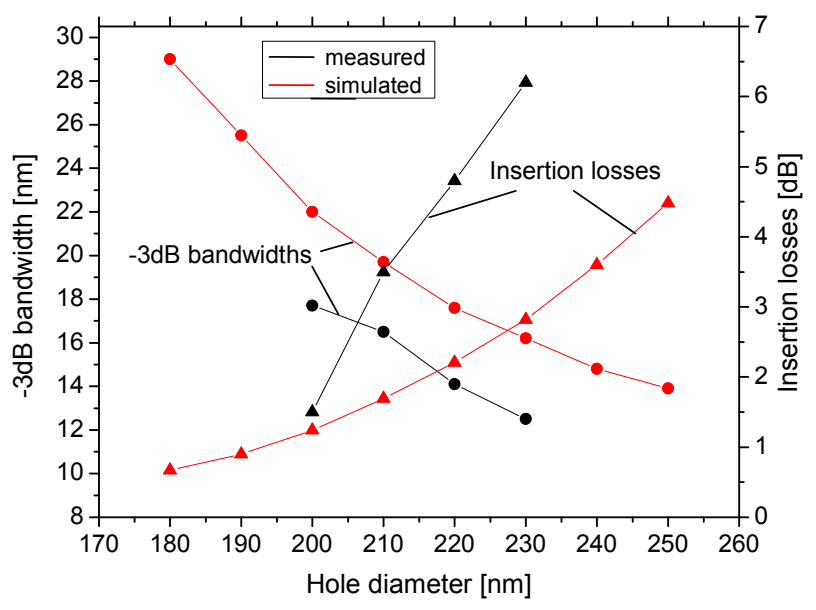

Figure 8. Measured and simulated $-3 \mathrm{~dB}$ bandwidths as well as insertion losses of the filters depending on the hole diameter for first order single cavity filters. The cavity length of the $3+3$ filters is $l_{c}=630 \mathrm{~nm}$.

Furthermore, the influence of hole diameter on the transmission band of the filters is investigated. Figure 8 shows the $-3 \mathrm{~dB}$ bandwidths as well as insertion losses of single cavity $3+3$ filters depending on the hole diameter for first order filters. For all filters, the cavity length is $1_{c}=630 \mathrm{~nm}$. Both, the measure and the simulated curves show a decay of the bandwidth with increasing the hole diameter. This bandwidth effect can be interpreted by the dielectric Fabry-Perot filter 
analogy, where the larger hole diameter is closer to the optimum ratio of hole diameter to lattice constant. But, radiation losses rapidly increase with increasing hole diameter. For hole diameters of $200 \mathrm{~nm}$ the measured und simulated loss values are comparable to each other. But for larger hole diameters, the measured values rise much stronger. That can be explained by the occurrence of corrugations at the waveguide edges mentioned in section 3, which introduce additional losses. Also the center-wavelength shifts if the hole diameter $d_{h}$ change. The wavelength shift was determined to be $\Delta \lambda / \Delta \mathrm{dh}=-1.8 \pm 0.2$ for the measured filters.

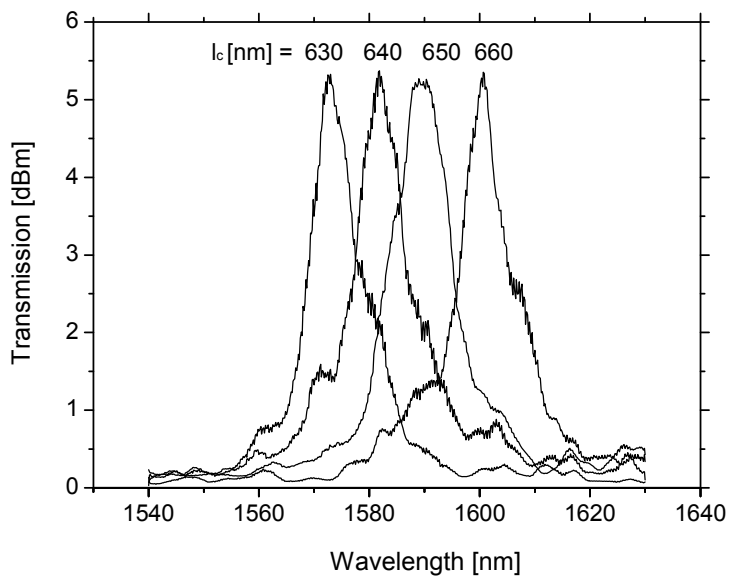

Figure 9. Filter transmission spectra for different cavity lengths $1_{c}$. $4+4$ filters with $200 \mathrm{~nm}$ hole diameter.

The cavity length $1_{c}$ mainly defines the filter-peak wavelength. Figure 9 shows transmission spectra of filters with different cavity lengths $1_{c}$. It is evident that an increasing cavity length will shift the filter peak to a longer wavelength. This behavior is also calculated for $4+4$ filters with $200 \mathrm{~nm}$ hole diameter and varied cavity length ranging from $1_{c}=610 \mathrm{~nm}$ to $660 \mathrm{~nm}$. The obtained wavelength shift per cavity length yields nearly constant $\Delta \lambda / \Delta \mathrm{l}_{\mathrm{c}}=1 \pm 0.6$ in the considered limited range of cavity lengths. About the same shift value has been observed in ${ }^{7}$.

\subsection{Higher order cavity filters}

In this section higher order photonic crystal cavities are investigated. A higher order cavity has a longer $1_{\mathrm{c}}$ which again gives a transmission peak at the desired wavelength. In a classical Fabry-Perot the spacer thickness has to be $d=m \lambda / 2 n$ to fulfill the conditions for resonance. Thereby, $n$ is the refractive index of the spacer material und $m$ is an integer. In our 1D photonic crystal cavities, $n$ has to be replaced by the effective refractive index of the SIO waveguide, which was calculated to $n_{\text {eff }}=2.40$. The formula does not apply for the first order, because the cavity length $1_{c}$ is measured form the center of the inner holes, respectively.
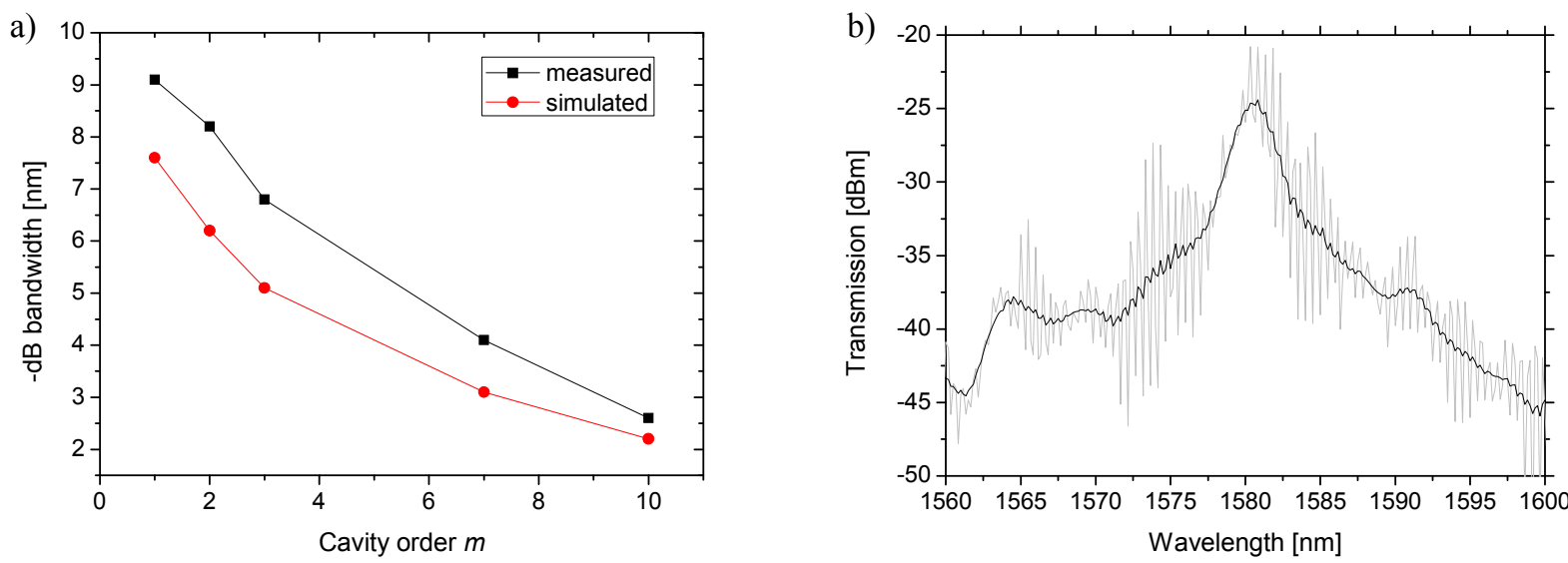

Figure 10. a) $-3 \mathrm{~dB}$ bandwidth depending on the cavity order $m$ for $4+4$ filters with hole diameter of $200 \mathrm{~nm}$. b) Transmission spectrum of a $10^{\text {th }}$ order single cavity filter with cavity length of $3537 \mathrm{~nm}$. The $-3 \mathrm{~dB}$ bandwidth is $2.4 \mathrm{~nm}$ corresponding to a quality factor of $\mathrm{Q}=660$. 
The cavity length of higher order filters can then be estimated by $1_{\mathrm{cH.O}}=1_{\mathrm{cl} . \mathrm{O}}+(m-1) \lambda / 2 n$. The integer $m$ gives the order number of the higher order cavities.

Figure 10a shows the $-3 \mathrm{~dB}$ bandwidth of the filter peaks for 5 different cavity orders corresponding to different cavity lengths, respectively. All filters consist of $4+4$ holes and have hole diameters of $200 \mathrm{~nm}$. The bandwidths of the filters decrease with the cavity order and start to saturate for high order numbers. The simulated bandwidths are somewhat smaller but showing the same trend.

Figure $10 \mathrm{~b}$ shows the transmission spectrum of a $10^{\text {th }}$ order single cavity filter with a cavity length of $3537 \mathrm{~nm}$. The $-3 \mathrm{~dB}$ bandwidth of this filter is $2.4 \mathrm{~nm}$. For a center-wavelength of $1580 \mathrm{~nm}$ this corresponds to a quality factor of Q $=660$. The insertion loss of this filter is $4.7 \mathrm{~dB}$.

\subsection{Multi-cavity filters}

Multi-cavity filters are useful to shape the transmission curve. Especially, the flat top shape and the strong decay in transmission at the edges of the filter curve make this kind of filter attractive for several applications, e.g. WDM. Higher order 1D photonics crystal cavities were already proposed in ${ }^{10}$.

Beside the single cavities, double and triple cavity filters are investigated in this work. All cavities inside of one filter have the same lengths. The bandwidths of selected multi-cavity filters are shown in table 1 . The cavity lengths of the filters are of first order and the outer mirrors consist of 3 holes. The $-3 \mathrm{~dB}$ bandwidth of the filters decrease with the number of cavities to about one half for the $3+6+6+3$ filter compared to the single cavity $3+3$ filter. A much stronger decay was observed for the $-10 \mathrm{~dB}$ bandwidths of the filters, also shown in table 1 . Here, the $-10 \mathrm{~dB}$ bandwidth of the $3+6+6+3$ filter is about one fourth compared to the single cavity $3+3$ filter. The simulated bandwidths are slightly larger, but show consistent behavior for in the different cavities.

Table 1. Simulated and measured $-3 \mathrm{~dB}$ as well as $-10 \mathrm{~dB}$ bandwidths depending on the number of cavities. The hole diameter was always $200 \mathrm{~nm}$ and the cavity length $l_{\mathrm{c}}=660 \mathrm{~nm}$.

\begin{tabular}{|c|c|c|c|c|}
\hline Cavity & $\begin{array}{c}-3 d B \text { bandwidth } \\
{[\mathrm{nm}]} \\
\text { measured }\end{array}$ & $\begin{array}{c}-3 \text { dB bandwidth } \\
{[\mathrm{nm}]} \\
\text { simulated }\end{array}$ & $\begin{array}{c}-10 \mathrm{~dB} \text { bandwidth } \\
{[\mathrm{nm}]} \\
\text { measured }\end{array}$ & $\begin{array}{c}-10 \mathrm{~dB} \text { bandwidth } \\
{[\mathrm{nm}]} \\
\text { simulated }\end{array}$ \\
\hline $3+3$ & 17.7 & 20 & 55.5 & 66.5 \\
\hline $3+6+3$ & 11 & 12.5 & 22 & 22.4 \\
\hline $3+6+6+3$ & 8.8 & 11.5 & 14.5 & 16.7 \\
\hline
\end{tabular}

Figure 11a shows the measured and figure $11 \mathrm{~b}$ the simulated transmission band of the $3+6+6+3$ multi-cavity filter given in table 1 . The cavity lengths of the filter are of first order and the outer mirrors consist of 3 holes. All holes have a diameter of $200 \mathrm{~nm}$. The measured and simulated filter curves show very similar shape. The measured insertion loss of the filter in figure $11 \mathrm{a}$ is $3.5 \mathrm{~dB}$. Compared to the single cavity $4+4$ filter in figure 6 , the filter contrast is more than $15 \mathrm{~dB}$ for wavelengths $10.6 \mathrm{~nm}$ off the filter peak wavelength.
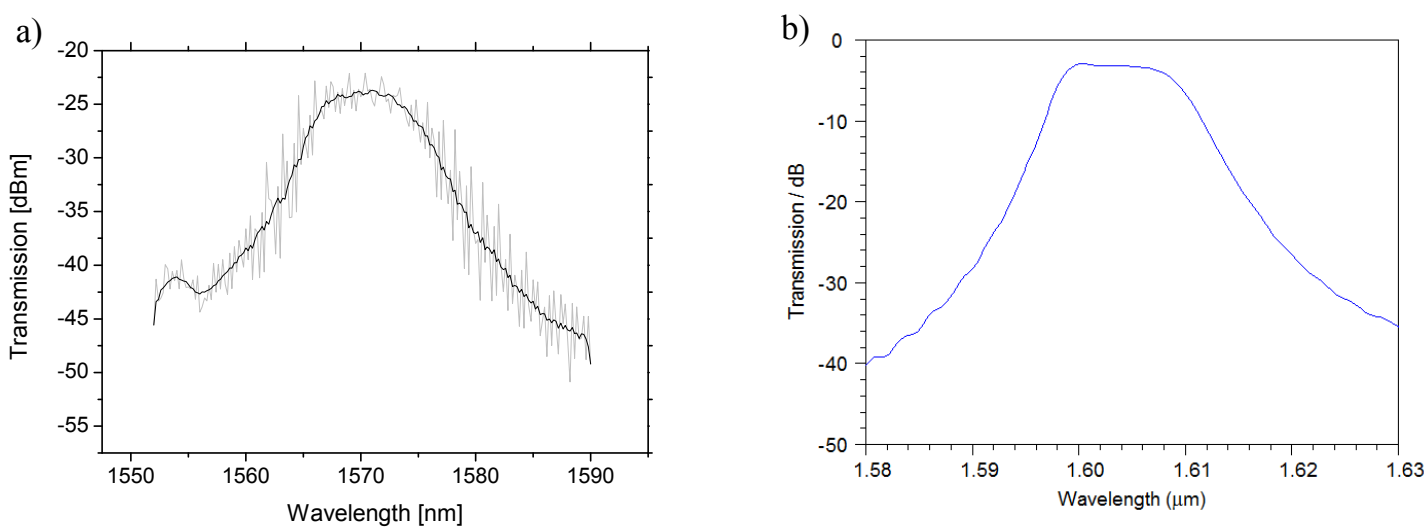

Figure 11. First order multi-cavity filter with $3+6+6+3$ holes. The hole diameter is $200 \mathrm{~nm}$. a) Measured filter curve with a $-3 \mathrm{~dB}$ bandwidth of $8.8 \mathrm{~nm}$ and a $-10 \mathrm{~dB}$ bandwidth of $14.5 \mathrm{~nm}$. b) Simulated filter curve with a $-3 \mathrm{~dB}$ bandwidth of $11.5 \mathrm{~nm}$ and a $-10 \mathrm{~dB}$ bandwidth of $16.7 \mathrm{~nm}$. 
Higher order multi-cavity filters were also investigated. Increasing the cavity order of a multi-cavity filter enables fine adjustment of the filter bandwidth ${ }^{11}$. In figure 12 a $10^{\text {th }}$ order $3+6+3$ filter is shown. With a $-3 \mathrm{~dB}$ bandwidth of $3.8 \mathrm{~nm}$ and a $-10 \mathrm{~dB}$ bandwidth of $12.9 \mathrm{~nm}$ the transmission band of this filter is much narrower than the first order $3+6+3$ filter given in table 1. The insertion loss of the filter was determined to $3.6 \mathrm{~dB}$.

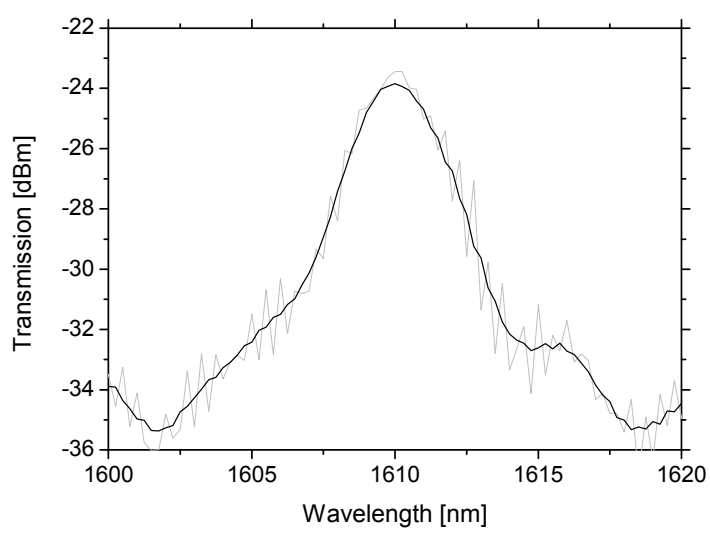

Figure 12. Transmission spectrum of a $10^{\text {th }}$ order multi-cavity filter with $3+6+3$ holes and $200 \mathrm{~nm}$ hole diameter. The $-3 \mathrm{~dB}$ bandwidth of the filter is $3.8 \mathrm{~nm}$ and the $-10 \mathrm{~dB}$ bandwidth is $12.9 \mathrm{~nm}$.

\section{SUMMARY}

Design, fabrication, and optical characterization of 1-D photonic crystal microcavities in SOI waveguides fabricated in a CMOS environment using a $248 \mathrm{~nm}$ DUV lithography have been presented. This paper mainly focused on wavelength filters for application in telecommunication systems around wavelengths of $1550 \mathrm{~nm}$.

The influence of the hole diameter, the cavity length, and number of holes on the transmission band of filters have been investigated. It was found, that, for the used fabrication technology, photonics crystals with hole diameters of 200nm are well suited as bandpass filters with moderate losses.

The transmission band of single as well as double and triple cavity filters have been investigated in this work. It was shown that multi-cavity filters enable more rectangular shape of the spectral filter curves. A first order multi-cavity filter with $3+6+6+3$ holes and $200 \mathrm{~nm}$ hole diameter has a $-3 \mathrm{~dB}$ bandwidth of $8.8 \mathrm{~nm}$ and a $-10 \mathrm{~dB}$ bandwidth of $14.5 \mathrm{~nm}$. Furthermore, a quality factor of $\mathrm{Q}=660$ is observed for a $10^{\text {th }}$ order single cavity filter with a cavity length of $3537 \mathrm{~nm}$. The insertion loss of this filter is $4.7 \mathrm{~dB}$. Finally, the transmission band of a higher order multi-cavity filter has been analyzed. Such filters enable fine adjustment of the filter bandwidth.

\section{ACKNOWLEGEMENT}

This work is supported by BMBF/VDI in the frame of project $13 \mathrm{~N} 9732$.

Thanks to Christian Meuer from Institut für Festkörperphysik of TU Berlin for his support.

\section{REFERENCES}

1. Izhaky, N., Morse, M. T., Koehl, S., Cohen, O., Rubin, D., Barkai, A., Sarid, G., Cohen, R. and Paniccia, M. J., "Development of CMOS-Compatible integrated silicon photonics devices", IEEE Journal of selected optics in quantum electronics, 12(6), 1688 (2006).

2. Reed, G., [Silicon Photonics: the state of the art], John Wiley \& Sons Ltd, England (2008).

3. Noda, S., Chutinan, A., and Imada, M., "Trapping and emission of photons by a single defect in a photonic bandgap structure", Nature (London) 407, 608 (2000).

4. Scheuera, J., Green, W. M. J., DeRose, G. A., and Yariv, A., "Lasing from a circular Bragg nanocavity with an ultrasmall modal volume", Appl. Phys. Lett. 86, 251101 (2005). 
5. Dumon, P., Bogaerts, W., Wiaux, V., Wouters, J., Beckx, S., Van Campenhout, J., Taillaert, D., Luyssaert, B., Bienstman, P., Van Thourhout, D. and Baets, R., "Low-loss SOI photonic wires and ring resonators fabricated with deep UV lithography", IEE photon. Technol. Lett., 16, 1328-1330 (2004).

6. Foresi, J. S., Villeneuve, P. R., Ferrera, J.,Thoen, E. R., Steinmeyer, G., Fan, S., Joannopoulos, J. D. Kimerling, L. C., Smith, H. I., \& Ippen, E. P., "Photonic-bandgap microcavities in optical waveguides", Nature, Papers 390 (6656), 143-135 (1997).

7. Kuhlow, B., Przyrembel, G., Schlüter, S., Fürst, W., Steingrüber, R., \& Weimann, Ch., "Photonic Crystal Microcavities in SOI Photonic Wires for WDM Filter Applications", Journal of Lightwave Technology, 25(1), 421-431 (2007).

8. Villeneuve, P. R., Fan, S., Joannopoulos, J. D., Lim, K.-Y., Petrich, G. S. , Kolodziejski, L. A. and Reif, R., "Air-bridge microcavities", Appl. Phys. Lett. 67 (2), 167-169, (1995).

9. Lefevre, H. C., "Single-mode fiber fractional wave devices and polarization controllers", Electronic Lett., 16 (20), 778-780, (1980).

10. Przyrembel, G., Kuhlow, B. and Schlüter, S., "Higher order PBG flat-top transmission waveguide filters in SOI", Eur. Conf. Optical Commun. (ECOC), 5, Tu1.1.6, Rimini, Italy (2003).

11. Przyrembel, G., Kuhlow, B. and Schlüter, S., "Flat-top transmission waveguide filter with multiple cavities," in Proc. Workshop and EOS Top. Meeting 2D Photonic Cryst., pp. (1-20), Ascona, Switzerland, Aug. 25-30, (2002). 